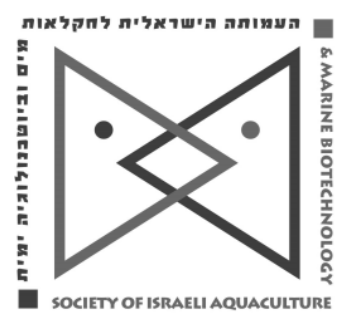

The IJA appears exclusively as a peer-reviewed on-line open-access journal at http://www.siamb.org.il/. To read papers free of charge, please register online at registration form.

Sale of $I J A$ papers is strictly forbidden.

\title{
Effects of Different Levels of Dietary Surfactin Supplementation on Intestinal Morphology, and Intestinal Microflora of Growth Retarded Marbled Eel Juveniles (Anguilla marmaorata)
}

\author{
Shao-Wei Zhai*, Xue-Hao Chen, Ming-Hao Wang \\ Fisheries College of Jimei University, Engineering Research Center of the \\ Modern Industry Technology for Eel, Ministry of Education, China
}

Keywords: surfactin; marbled eel; intestinal morphology; intestinal microflora

\begin{abstract}
This 70 day trial was conducted to investigate the effects of surfactin on intestinal morphology and intestinal microflora of growth retarded marbled eels (Anguilla marmorata). A total of six hundred and forty marbled eels at elver stage were randomly allocated to 4 treatment groups on the basis of body weight. There were 4 replicate tanks in each treatment group with 40 fish per tank. Four diets were formulated with four levels of dietary surfactin: $0,25,50$, and $100 \mathrm{mg} / \mathrm{kg}$, respectively. Fish were fed twice daily to apparent satiation for 70 days. Retarded marbled eels fed diets with surfactin supplementation had enhanced mucosa folds in their intestines $(P<0.05)$. It seems that the quantity of mucosa folds increased with the increasing surfactin levels in the diet. Dietary surfactin supplementation decreased the $E$. coli population significantly $(P<0.05)$, and increased the Lactobacillus population significantly $(P<0.05)$, but had no effect on total bacterial population in intestines of marbled eels $(P>0.05)$. Results of the present study indicate that surfactin improves intestinal function in retarded elvers of marbled eels.
\end{abstract}

\footnotetext{
* Corresponding author. Tel: +86-592-6181420, Fax: +86-592-6181476. email: shaoweizhai@hotmail.com
} 


\section{Introduction}

Eel is a commercially valuable important aquaculture species, and important to the freshwater aquaculture industry in Southeast Asia (Huang et al., 2016). In general, under farming conditions, individual variation in eel growth is considerable, and frequent grading is needed to keep the sizes separate. Eels at elver stage are usually graded into three sizes, big, normal, and small size stock. Growth of about $10-30 \%$ of eel in cultivation is retarded in the cultivation process; this may be due to feeding mismanagement in the early stages, a harmful environment, diseases, or parasites. Under these circumstances, fish are small, some have low feed intake resulting in very slow growth rate with nearly no weight gain, however feed intake in normal individuals from the same initial stock is higher, and body weight of these fish may be ten or more times that of the retarded individuals (Wang et al., 1999; Huang, 2012). Digestive enzyme activity in the intestines of retarded eels was reported to be significantly lower than in normal eels, so certain feed supplements might promote growth of retarded eels (Huang, 2012). Some antibiotic drugs and hormones have been used to promote the growth of retarded eels, however the safety of these solutions is questionable (Siwicki et al., 2013; Ni et al., 1992; Wang et al., 1999).

Surfactin, an antimicrobial lipopeptide from secondary metabolites produced by several strains of Bacillus subtilis, is a cyclic lipoheptapeptide containing seven amino acids and a $\beta$-hydroxyl fatty acid $\left(\mathrm{C}_{13}-\mathrm{C}_{15}\right)$ (Seydlova and Svobodova, 2008; Chen et al., 2015). It has been characterized as anti-bacterial, anti-viral, anti-fungal, a biosurfactant, immunoadjuvant, anti-tumor, antimycoplasma, and as having hemolytic activity, all of which are important in health care and biotechnological application (Seydlova and Svobodova,2008; Liu et al., 2010; Chen et al., 2015). Surfactin, as an antimicrobial peptide (AMPs), has received much attention in feed supplementation in some fish species, including tilapia Oreochromis niloticus, (Shi, 2015; Zhai et al., 2016a), marbled eel Anguilla marmorata, (Zhai et al., 2016b), grouper Epinephelus coioides, (Sun, 2016; Zhai et al., 2017), Pacific white shrimp (Litopenaeus vannamei) (Shi et al., 2014), and Chinese soft-shelled turtle Pelodiscus sinensis, (Zhai et al., 2015). Improved growth performance has been reported from dietary surfactin supplementation in tilapia and grouper; this is perhaps attributed to increased nutrient availability for absorption via suppression of growth and metabolic activities of harmful gut microflora with simultaneous alteration in intestinal morphology, intestinal epithelium thickness, and epithelial cell turnover (Shi, 2015; Sun, 2016; Zhai et al., 2016a). As it is a natural and safe product of Bacillus subtilis fermentation (Seydlova and Svobodova, 2008; Chen et al., 2015), a recent study in our lab reported that dietary surfactin supplementation had beneficial effects on growth performance of retarded marbled eels (Zhai et al., 2016b). Little information is available about the effect of dietary surfactin supplementation on intestinal health of eels. The purpose of the present study was to investigate the effect of dietary surfactin supplementation on intestinal morphology and intestinal microflora of retarded marbled eels at elver stage.

\section{Materials and Methods}

Animal acquisition and maintenance. Nine hundred and sixty retarded marbled eels at elver stage were purchased at the Development Center for Aquatic Animals in Putian (China). Small size stock fish after one year of cultivation were selected. The fish ( 80 per tank) were cultured in 16 circular PVC tanks and in a recirculating water system. After a four-week adaption period, 40 fish were left in each tank as the trial animals. The tanks $(2 \mathrm{~m} \times 2 \mathrm{~m} \times 1.5 \mathrm{~m})$ were equipped with bio-filters, UV lights, heaters, and chillers (Huixin Marine Science and Technology Development Co., LTD, Dalian, China). The water temperature was kept at $28-30^{\circ} \mathrm{C}$. Water quality variables were: salinity $0.2 \%$; pH 6.68.0; dissolved oxygen $>6.3 \mathrm{mg} / \mathrm{L}$; total ammonia nitrogen $0.2-0.5 \mathrm{mg} / \mathrm{L}$; nitrite nitrogen levels $<1.0 \mathrm{mg} / \mathrm{L}$. The water quality was monitored twice weekly with a multiparameter photome (HI9804N, HANNA, Baranzate, MI, Italy). Water exchange rate was $10 \%$ per day. The photoperiod was maintained at $12 \mathrm{~L}$ : $12 \mathrm{D}$.

The fish were fed a commercial powder diet produced by Zhengyuan Feed Co., Ltd., Fujian, China. The commercial powder diet contained $47 \%$ protein, $4 \%$ lipids, $3 \%$ crude 
fiber, $17 \%$ ash, and $10 \%$ moisture. The diet was added to 1.2 volume water and mixed into dough. The dough was placed on a feeding table and served to the eels. During the adaptation and trial periods, the fish were fed to apparent satiation twice daily (at 8:00 $\mathrm{h}$ and 18:00 h); any uneaten feed was siphoned out $1 \mathrm{~h}$ after feeding and dried. The total amount of daily consumed feed was calculated by subtracting the uneaten food from the eaten food.

Experimental design, diets, and fish management. Six hundred and forty retarded marbled eels with an initial average body weight of $3.02 \pm 0.02 \mathrm{~g}$ were randomly selected and divided into four treatment groups with four replicates in each group, and 40 fish in each replicate. All treatment groups were fed the diets with the respective surfactin levels being 0 (control group), 25, 50, and $100 \mathrm{mg} / \mathrm{kg}$. The surfactin (content $>80 \%$ ) was provided by Fujian Zhengyuan Feed Co., Ltd., Putian, China. The trial lasted for 70 days.

Sample collection and analysis. At the end of the trial, all fish were starved for $24 \mathrm{~h}$ to empty the gut, and six fish were sampled at random from each replicate. Three fish from each tank were anesthetized with $50 \mu \mathrm{l} / \mathrm{L}$ of eugenol oil suspension, and then killed on an ice bath for intestine samples. Segments of intestines of those three fish from each tank, with an approximative length of $2 \mathrm{~cm}$ were collected for morphology analysis. About $0.5 \mathrm{~cm}$ segments were used and washed by phosphate buffer saline solution, and fixed in Bouin solution. After fixation, the segments were successively dehydrated in ethanol, equilibrated in xylene, and embedded in paraffin wax according to the standard histological procedures (Hu et al., 2015; Qi et al., 2016). The samples were then sliced into $6 \mu \mathrm{m}$ longitudinal sections following the axis of intestine lumen and stained with Hematoxylin-Eosin (H\&E). The intestine slides were examined and photographed under an Olympus microscope (BX80, Japan). The height of mucosa fold (MF) was measured by the method described by Zhai et al. (2016a). Five measurements were conducted for height of MF per intestinal slice.

Another three fish from each tank were sacrificed and the skin washed with $75 \%$ ethanol to reduce the number of incidental organisms before opening the ventral cavity surface with sterile scissors. The intestine of each fish was washed with phosphate buffer solution three times to clean the contents, and then transferred to a sterile test tube. About $0.5 \mathrm{~g}$ of intestine sample were weighed, diluted 10-fold with sterile $9 \mathrm{~g}$ phosphate buffer solution, and homogenized in a mortar $(5000 \mathrm{r} / \mathrm{min})$. After homogenizing, $10 \mathrm{ml}$ with saline, the homogenate was serially diluted ten-fold in sterile saline. $0.1 \mathrm{ml}$ of each dilution was spread onto the selective agars of MRS, EMB, and TTC (Huankai Microbial Technology Co., Ltd of Guangzhou, China), which were used to determine total bacteria, E. coli, and Lactobacillus, respectively. Plates were incubated at $28^{\circ} \mathrm{C}$ for 48 hours. Following incubation, plates containing 30 to 300 colonies were used to calculate bacterial population results, recorded as CFU/g wet intestine. All microbiological analyses were performed in duplicate and the average values were used for statistical analysis.

Statistical analysis. The results are presented as means \pm SD of four replicates. Statistical analysis was performed with SPSS 11.5 statistical software (SPSS, Chicago, IL, USA). Data from each treatment group were subjected to one-way analysis of variance (ANOVA). When overall differences were significant $(P<0.05)$, Duncan's multiple range test was used to compare the mean values among the treatment groups.

\section{Results}

Mucosa fold height. Height of mucosa folds of different marbled eels groups are shown in table 1 and figure 1 . The height of MF of fish fed surfactin were significantly greater than that of the control group $(P<0.05)$. There were no significant differences between the groups $(P>0.05)$. As shown in figure 1 , the density of MF of fish fed diet supplemented surfactin were higher than in the control group, and quantity of MF within the same field of view increased with the increasing surfactin levels in diets. 
Table 1. Effects of surfactin on mucosa fold height of intestine of growth retarded marbled eel

\begin{tabular}{ll}
\hline Surfactin levels & Height of $M F, \mu m$ \\
\hline $0($ Control group) & $327.19 \pm 18.44^{\mathrm{a}}$ \\
$25 \mathrm{mg} / \mathrm{kg}$ & $415.11 \pm 9.28^{\mathrm{b}}$ \\
$50 \mathrm{mg} / \mathrm{kg}$ & $420.62 \pm 13.25^{\mathrm{b}}$ \\
$100 \mathrm{mg} / \mathrm{kg}$ & $418.28 \pm 15.78^{\mathrm{b}}$ \\
\hline
\end{tabular}

ab Values within the same column without the same superscript were significantly different at $P<0.05$ level.

$\mathrm{MF}=$ Mucosa fold

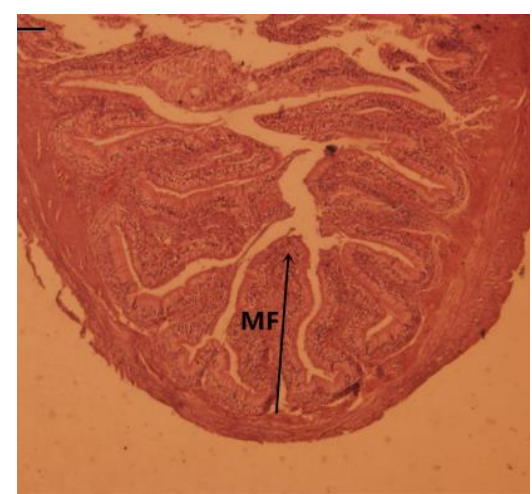

a

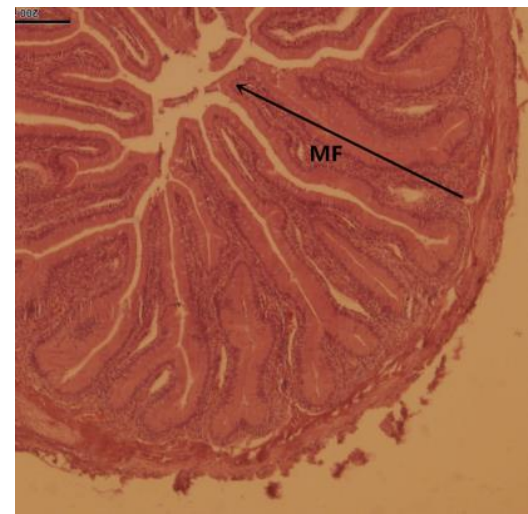

C

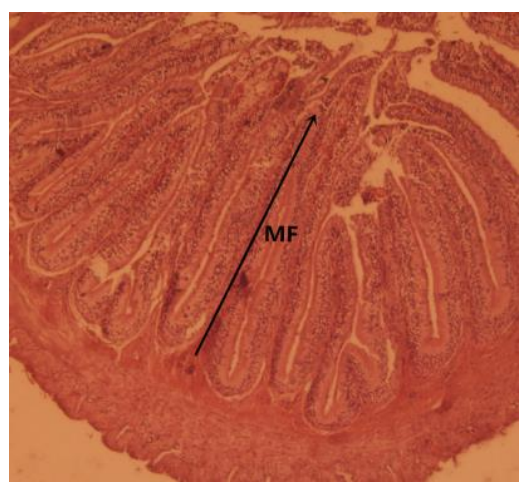

b

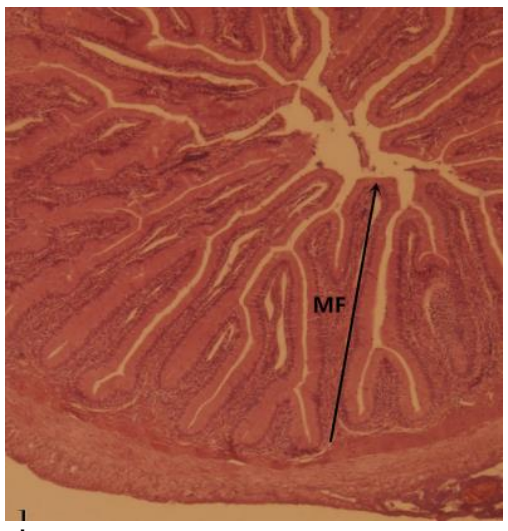

d

Figure1. Tissue slices of intestine of growth retarded marbled eel fed diets with different surfactin levels.

$\mathrm{a}, \mathrm{b}, \mathrm{c}$, and d show the intestine of fish fed diets with $0,25,50$ and $100 \mathrm{mg} / \mathrm{kg}$ surfactin. Arrows indicate the measurement position of fold height.

Staining: $\mathrm{H}$ and $\mathrm{E}$. Magnification $\times 200$. MF: mucosa fold.

Intestinal microflora. Table 2 presents the results of intestinal microflora of growth retarded marbled eel fed diets with different levels of surfactin. The total bacteria populations were similar among all the groups $(P>0.05)$. Compared with control group, the eel fed diets with surfactin supplementation had a lower population of $E$. coli $(\mathrm{P}<0.05)$, with the lowest value of $E$. coli population in $25 \mathrm{mg} / \mathrm{kg}$ surfactin group. The Lactobacillus population increased significantly with dietary $25 \mathrm{mg} / \mathrm{kg}$ surfactin supplementation $(P<0.05)$, and no significant difference was found between other two surfactin groups $(P<0.05)$. 
Table 2. Effects of surfactin on bacterial populations ( $\log 10 \mathrm{CFU} / \mathrm{g}$ ) of growth retarded marbled eel

\begin{tabular}{llll}
\hline Surfactin levels & Total & E. coli & Lactobacillus \\
\hline 0 (Control group) & $8.19 \pm 0.12$ & $7.32 \pm 0.06^{\mathrm{c}}$ & $6.93 \pm 0.12^{\mathrm{a}}$ \\
$25 \mathrm{mg} / \mathrm{kg}$ & $8.21 \pm 0.12$ & $6.86 \pm 0.12^{\mathrm{a}}$ & $7.24 \pm 0.04^{\mathrm{b}}$ \\
$50 \mathrm{mg} / \mathrm{kg}$ & $8.22 \pm 0.07$ & $7.07 \pm 0.04^{\mathrm{b}}$ & $6.84 \pm 0.09^{\mathrm{a}}$ \\
$100 \mathrm{mg} / \mathrm{kg}$ & $8.24 \pm 0.10$ & $7.10 \pm 0.02^{\mathrm{b}}$ & $6.82 \pm 0.06^{\mathrm{a}}$ \\
\hline
\end{tabular}

abcValues within the same column without the same superscript were significantly different at $\mathrm{P}$ $<0.05$ level.

\section{Discussion}

In the present study, the supplementation of surfactin to diets of growth retarded marbled eel resulted in greater height and density of MF in intestines of fish. Similar results were also reported in a previous study of tilapia (Zhai et al., 2016a). The intestinal morphology is indicative of the gut health in fish. Increased MF height and density suggests an increase of the surface area capable of greater absorption of available nutrients, epithelial turnover, and activation of cell mitosis (Caspary, 1992; Fan et al., 1997; Samanya and Yamauchi, 2002). There is increased evidence that AMPs including surfactin maintain intestinal epithelial barrier integrity by stimulation of mucus synthesis to protect the underlying epithelium from mechanical, chemical, enzymatic, and microbial damage, increasing the synthesis of tight junction proteins and reparation of the intestinal barrier by stimulating cell proliferation and promoting the production of extracellular matrix proteins. AMPs can also function as potent immune regulators and protect the intestinal surface (Wang et al., 2015).

It is well known that AMPs can be beneficial to host animals by improving intestinal balance and creating gut microecological conditions that suppress harmful microorganisms and by favoring beneficial microorganisms (Wang et al., 2015). In this study, dietary supplementation of surfactin reduced harmful microflora like E.coli and increasing beneficial microflora like Lactobacillus. This was similar to the number of $E$. coli in tilapia intestine where the number of Lactobacillus increased significantly with surfactin supplementation (Zhai et al., 2016a). Surfactin has broad spectrum antimicrobial activity against bacterial, fungi, and viral pathogens. It was found that surfactin could induce 'pore' formation of biological membranes underlying the antibiotic and other important biological actions of this bacterial lipopeptide (Seydlova and Svobodova, 2008). It interacts with the lipid membrane in three different ways. It acts as a mobile cation carrier, forms cationic channels, and destroys the membrane via a detergent effect (Seydlová et al., 2013). Surfactin can function in vivo but is not detrimental to host cells. The partial selectivity of AMPs for prokaryotic cells may be due to the absence of cholesterol in the bacterial cell membranes. Because cholesterol is well known to induce condensation of phospholipid bilayers, AMPs might be prevented from penetrating into the cytoplasmic membrane of eukaryotic cells (Lai and Gallo, 2009). In addition, the hydrophobicities of AMPs are generally too low to effectively bind to zwitterionic phospholipids, avoiding toxicity to the host (Matsuzaki, 1999).

Our study demonstrated the beneficial effects of dietary surfactin supplementation on morphology and microflora in the intestine of growth retarded marbled eels. These results explain the reason why growth performance and some digestive enzyme activity in fish intestine were significantly improved by surfactin supplemented in the diet (Zhai et al., 2016b). Our previous results together with those in present trial, show that these improvements in eels might be due to increased nutrient availability for absorption via suppression of growth and metabolic activities of harmful gut microflora with simultaneous alteration in intestinal morphology (Shi, 2015; Sun, 2016; Zhai et al., 2016a).

In this study, $25 \mathrm{mg} / \mathrm{kg}$ of surfactin in growth retarded marbled eel diet resulted improved intestinal health status. In the previous tilapia study, dietary $50 \mathrm{mg} / \mathrm{kg}$ surfactin might ameliorate intestinal health status (Zhai et al., 2016a). In another 
study, $100 \mathrm{mg} / \mathrm{kg}$ surfactin had beneficial effects on the intestinal or liver health of orange-spotted grouper juveniles (Sun 2016). Surface activity of surfactin might be different on cell membranes of animal species (Shi, 2015; Zhai et al., 2016a). The biological function of surfactin is dependent on the surface activity in cell membrane, and surfactin concentration is critical for its effect on the cell membrane (Seydlova and Svobodova, 2008; Deleu et al., 2013; Chen et al., 2015). Further study is needed to clarify in detail the mechanism of different surfactin doses and the biological effect on different aquatic animal cell membranes.

Changes of morphology and microflora in the intestine of growth retarded marbled eels reported in the present study indicate that surfactin, as one of AMPs, could be of great importance in maintaining normal gut homeostasis. This provides new information regarding the potential for using surfactin in fish feed as an intestinal health promoter.

In conclusion, this study demonstrated that supplementary surfactin in diets had beneficial effects on intestinal morphology and intestinal microflora of growth retarded marbled eels at elver stage.

\section{Acknowledgements}

This study was supported by China Agriculture Research System (CARS-46) and Special Project of International Science and Technology Cooperation and Exchange of Ministry of Science and Technology (No.2014DFT30150).

\section{References}

Caspary W.F., 1992. Physiology and pathophysiology of intestinal absorption. Am. J. Clin. Nutr., 55:299-308.

Chen W.C., Juang R.S., Wei Y.H., 2015. Applications of a lipopeptide biosurfactant, surfactin, produced by microorganisms. Biochem. Eng. J., 103:158-169.

Deleu M., Lorent J., Lins L., Brasseur R., Braun N., EI K.K., Nylander T., Dufrene Y.F., Mingeot-Leclercq M. P., 2013. Effects of surfactin on membrane models displaying lipid phase separation. BBA-Biomembranes, 1828(2):801-815.

Fan Y., Croom J., Christensen V., Black B., Bird A., Daniel L., McBride B., Eisen E., 1997. Jejunal glucose uptake and oxygen consumption in turkey poults selected for rapid growth. Poult. Sci., 76:1738-1745.

Hu H., Mai K., Zhang Y., Ai Q., Xu W., Zhang W., Li Y., Liu J., 2015. Effects of dietary xylan on growth performance, digestive enzyme activity and intestinal morphology of juvenile turbot (Scophthalmus maximus L.). Isr. J. Aquacult.Bamidgeh, IJA_67.2015.1115, 10 pages.

Huang C.T., Chiou J.T., Khac H.T., Hsiao Y.J., Chen S.C., 2016. Improving the management of commercial giant mottled eel Anguilla marmorata aquaculture in Taiwan for improved productivity: a bioeconomic analysis. Fish. Sci., 82(1):95-111.

Lai Y. and Gallo R.L., 2009. AMPed up immunity: How antimicrobial peptides have multiple roles in immune defense. Trends Immunol., 30(3):131-141.

Liu J., Zou A.H, Mu B.Z., 2010. Surfactin effect on the physicochemical property of PC liposome.Colloids and Surfaces A: Physicochem. Eng. Aspects, 361:90-95.

Matsuzaki K., 1999. Why and how are peptide-lipid interactions utilized for selfdefense? Magainins and tachyplesins as archetypes. Biochim. Biophys. Acta, 1462(12): $1-10$.

Ni X., Cai W.Y., Zhang T.J., 1992. Study on advancing growth and rejuvenation of ossified eel when adding medicament such as hormones in feed. Fishery Sci., 11(3):14-18.

Qi C.L., Lin H.Z., Huang Z., Zhou C.P., Wang Y., Wang J., Niu J., Zhao S.Y., 2016. Effects of dietary arachidonic acid levels on growth performance, whole-body proximate composition, digestive enzyme activities and gut morphology of juvenile golden pompano trachinotus. Isr. J. Aquacult.-Bamidgeh, IJA_68.2016.1275, 9 pages.

Samanya, M. and Yamauchi K., 2002. Histological alterations of intestinal villi in 
chickens fed dried Bacillus subtilis var. natto. Comp. Biochem. Physiol., 133:95-104.

Seydlová G. and Svobodova J., 2008. Review of surfactin chemical properties and the potential biomedical applications. Cent. Eur. J. Med., 3(2):123-133.

Seydlová G., Fišer R., Cabala R., Kozlík P., Svobodová J., Pátek M., 2013. Surfactin production enhances the level of cardiolipin in the cytoplasmic membrane of bacillus subtilis. Biochimica Et Biophysica Acta, 1828:2370-2378.

Shi G., Sun L.J, Wang Y.L., Liu H.M., Xu D.F., Nie F.H., Liu Y., Ye R.Y., 2014. Effects of NT-6 antimicrobial lipopeptide on growth performance of Litopenaeus vannamei and Vibrio number during breeding process. Guangdong J. Agr. Sci., 41(12):119-122,137.

Shi Q.C., 2015. The effects of dietary surfactin supplementation on the growth and intestinal health of GIFT strain of Nile tilapia (Oreochromis niloticus). Degree Diss., Jimei University, Xiamen, China.

Siwicki A.K., Lepa A., Robak S., Kazuń K., Kazuń B., Głąbski E., 2013. Effects of methisoprinol on the innate immunity parameters in intensive rearing system of European eel (Anguilla anguilla). Isr. J. Aquacult.-Bamidgeh, IJA_66.2014.946, 6 pages.

Sun X.W., 2016. Effects of dietary surfactin supplementation on growth performance, lipid metabolism and liver health status of juvenile grouper (Epinephelus Coioides). Degree Diss., Jimei University, Xiamen, China.

Wang S., Thacker P.A., Watford M., Qiao S., 2015. Functions of antimicrobial peptides in gut homeostasis. Curr. Protein Pept. Sci., 16:582-591.

Wang X.H., Chen S.J., Xu L.M., 1999. Effect of flavomycin on growth retarded European eel. China Fish., (1):28-29.

Zhai S.W., Shi Q.C., Chen X.H., 2016a. Effect of dietary antimicrobial peptidessurfactin supplementation on parameters of intestinal health indices of genetically improved farmed tilapia (GIFT, Oreochromis niloticus). Acta Hydrobiologica Sinica, 40:823-829.

Zhai S.W., Shi Q.C., Chen X.H., 2016b.Effects of dietary surfactin supplementation on growth, digestive enzymes activities and antioxidant potential in intestine of growth retarded marbled eel (Anguilla marmorata) at elver stage. Isr. J. AquacultBamidgeh., IJA_68.2016.1282, 7 pages.

Zhai S.W., Shi Q.C., Sun P.X., Lu P., Guo Q.X., 2015. Effect of dietary surfactin supplementation on weight gain rate and blood biochemical parameters of juvenile Chinese soft-shelled turtle (Pelodiscus sinensis). Feed China, (22):39-41.

Zhai S.W., Sun X.W., Chen X.H., 2017. Effects of antimicrobial peptides surfactin administration on growth performance, intestinal digestive enzymes activities and some serum biochemical parameters of orange-spotted grouper (Epinephelus coioides) juveniles. Isr. J. Aquacult.-Bamidgeh, IJA_69.2017.1380, 7 pages. 